\title{
Rhodium-Catalyzed Organothio Exchange Reaction of $\alpha$-Organothioketones with Disulfides
}

\author{
Mieko Arisawa, ${ }^{a}$ Fumihiko Toriyama, ${ }^{a}$ and Masahiko Yamaguchi ${ }^{*}, a, b$ \\ ${ }^{a}$ Department of Organic Chemistry, Graduate School of Pharmaceutical Sciences, Tohoku University; Aoba, Sendai \\ 980-8578, Japan: and ${ }^{b}$ WPI Research Center, Advanced Institute for Materials Research, Tohoku University; Sendai \\ 980-8577, Japan. Received June 5, 2010; accepted July 6, 2010; published online July 8, 2010
}

$\mathrm{RhH}\left(\mathrm{PPh}_{3}\right)_{4}$ and 1,2-bis(diphenylphosphino)ethane (dppe) catalyzed the organothio exchange reaction of $\alpha$-organothioketones and organic disulfides. The reaction was affected by the structure of the substrate: $\alpha$-phenylthio and $\alpha$-alkylthio aryl ketones reacted effectively with diaryl and dialkyl disulfides; $\alpha$-phenylthio dialkyl ketones reacted with diaryl disulfides but not with dialkyl disulfides; diaryl disulfides with electron-donating $p$-substituents were more reactive than those with electron-withdrawing $p$-substituents.

Key words rhodium catalyst; organothio exchange reaction; thioketone; disulfide

We have been investigating transition-metal-catalyzed methods for the synthesis and transformation of organosulfur compounds. ${ }^{1,2)}$ During our investigation, we developed catalytic cleavage reactions of $\mathrm{C}-\mathrm{S}$ bonds. We reported the equilibrating organothio exchange reaction of 1-(alkylthio) alkynes $^{3)}$ and thioesters ${ }^{4)}$ with organic disulfides. The reactions can be regarded a method of synthesizing organosulfur compounds from other organosulfur compounds. In addition, note that, in these organothio exchange reactions, novel organorhodium intermediates were generated. It was expected that the metal-catalyzed $\mathrm{C}-\mathrm{S}$ bond cleavage reactions could be used for the transformation of organosulfur compounds. It was therefore considered interesting to develop such an organothio exchange reaction for various organosulfur compounds. In this study, the organothio exchange reaction of $\alpha$-organothioketones was examined (Chart 1). This reaction is a convenient method of synthesizing various $\alpha$ keto sulfides from a single organosulfur compound without using base or reactive sulfenylating reagents. ${ }^{5-13)}$ It is likely that the oxidative addition of thioketones to the rhodium complex provides the $\mathrm{C}-\mathrm{Rh}-\mathrm{SR}$ intermediates, which undergo alkylthio exchange forming other $\mathrm{C}-\mathrm{Rh}-\mathrm{SR}^{\prime}$ complexes. $\alpha$ Alkylthio exchange ketones are liberated by the reductive elimination with regeneration of rhodium catalyst.

When a mixture of $\alpha$-(phenylthio)acetophenone and bis ( $m$-methoxyphenyl) disulfide (3 eq) in tetrahydrofuran (THF) was heated at reflux for $1-2 \mathrm{~h}$ in the presence of $\mathrm{RhH}\left(\mathrm{PPh}_{3}\right)_{4}(1 \mathrm{~mol} \%)$ and 1,2-bis(diphenylphosphino) ethane (dppe) ( $2 \mathrm{~mol} \%), \alpha$-( $m$-methoxyphenylthio)acetophenone was obtained with $84 \%$ yield (Table 1 , entry 1 ). The yield decreased to $74 \%$, when 2 eq of the disulfide was used. The exchange product was not obtained in the absence of dppe, and the use of several other bidentate ligands revealed

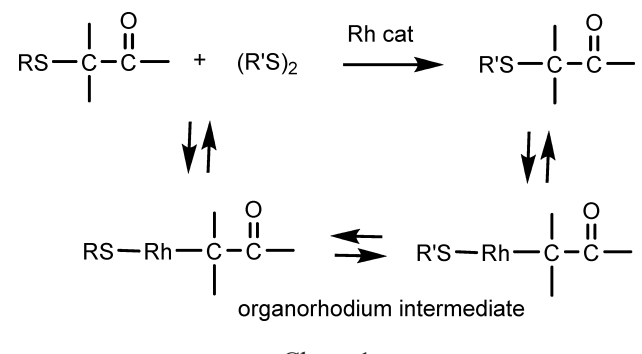

the high efficiency of dppe for this reaction: bis (diphenylphosphino)methane (dppm), not detected; 1,3,-bis (diphenylphosphino)propane (dppp), trace; cis-1,2-(diphenylphosphino) ethylene (dppv), 48\%; 1,2-bis (diphenylphosphino)benzene (dppBz), trace; 1,1'-bis (diphenylphosphino)ferrocene (dppf), not detected. The monodentate phosphine ligands $\left(p-\mathrm{ClC}_{6}\right.$ $\left.\mathrm{H}_{4}\right)_{3} \mathrm{P}$ and $\left(p-\mathrm{MeOC}_{6} \mathrm{H}_{4}\right)_{3} \mathrm{P}$ were not effective. The structure of the rhodium complex was also critical: the olefin complex $\mathrm{Rh}(\mathrm{acac})\left(\mathrm{CH}_{2}=\mathrm{CH}_{2}\right)$ showed catalytic activity; the Wilkinson complex $\mathrm{RhCl}\left(\mathrm{PPh}_{3}\right)_{3}$ and the cation complex [Rh(cod) $\left.\left(\mathrm{PPh}_{3}\right)_{2}\right] \mathrm{PF}_{6}$ were not effective; the palladium complexes $\mathrm{Pd}_{2}(\mathrm{dba})_{3}$ and $\mathrm{Pd}\left(\mathrm{PPh}_{3}\right)_{4}$ did not show catalytic activity.

Various combinations of ketonic substrates and disulfides were subjected to the reaction. $\alpha$-Phenylthio aryl ketones underwent a smooth arylthio exchange with diaryl disulfides (Table 1, entries 1, 2, 4-7). Dialkyl disulfides also reacted under the conditions giving $\alpha$-alkylthio ketones (entries 3, $8-10)$. The $p$-methoxy substituent on the aromatic ketones did not affect the reaction (entry 4 ). The $\beta$-elimination reaction to form unsaturated ketones was not observed in the reactions of propiophenone and butyrophenone derivatives (entries 5-10). The reaction of a tertiary sulfide, $\alpha$ (phenylthio)isobutyrophenone, however, gave a very low yield of the exchange product.

The reaction of $\alpha$-(octylthio)propiophenone was examined

Table 1. Rhodium-Catalyzed Organothio Exchange Reaction of $\alpha$ Phenylthio Aryl Ketones with Disulfides

\begin{tabular}{|c|c|c|c|c|}
\hline \multirow[b]{2}{*}{ Entry } & $\begin{array}{r}+\quad\left(R^{2} S\right) \\
3 \text { eq }\end{array}$ & \multicolumn{2}{|c|}{$\begin{array}{l}\mathrm{RhH}\left(\mathrm{PPh}_{3}\right)_{4}(1 \mathrm{~mol} \%) \\
\mathrm{dppe}(2 \mathrm{~mol} \%)\end{array}$} & $\underbrace{\mathrm{O}}_{\mathrm{R}^{1}}$ \\
\hline & $\mathrm{Ar}$ & $\mathrm{R}^{1}$ & $\mathrm{R}^{2}$ & Yield (\%) \\
\hline 1 & $\mathrm{Ph}$ & $\mathrm{H}$ & $m-\mathrm{MeOC}_{6} \mathrm{H}_{4}$ & 84 \\
\hline 2 & & & $p-\mathrm{MeC}_{6} \mathrm{H}_{4}$ & 83 \\
\hline 3 & & & $\mathrm{MeO}\left(\mathrm{CH}_{2}\right)_{3}$ & 82 \\
\hline 4 & $p-\mathrm{MeOC}_{6} \mathrm{H}_{4}$ & & $m-\mathrm{MeOC}_{6} \mathrm{H}_{4}$ & 85 \\
\hline 5 & $\mathrm{Ph}$ & $\mathrm{Me}$ & $m-\mathrm{MeOC}_{6} \mathrm{H}_{4}$ & 84 \\
\hline 6 & $p-\mathrm{MeC}_{6} \mathrm{H}_{4}$ & & $m-\mathrm{MeOC}_{6} \mathrm{H}_{4}$ & 83 \\
\hline 7 & $\mathrm{Ph}$ & Et & $m-\mathrm{MeOC}_{6} \mathrm{H}_{4}$ & 83 \\
\hline 8 & & & $\mathrm{MeO}\left(\mathrm{CH}_{2}\right)_{3}$ & 82 \\
\hline 9 & & & cyclo- $\mathrm{C}_{5} \mathrm{H}_{11}$ & 79 \\
\hline 10 & & & cyclo- $\mathrm{C}_{6} \mathrm{H}_{13}$ & 79 \\
\hline
\end{tabular}


Table 2. Rhodium-Catalyzed Organothio Exchange Reaction of $\alpha$ (Octylthio)propiophenone with Disulfides

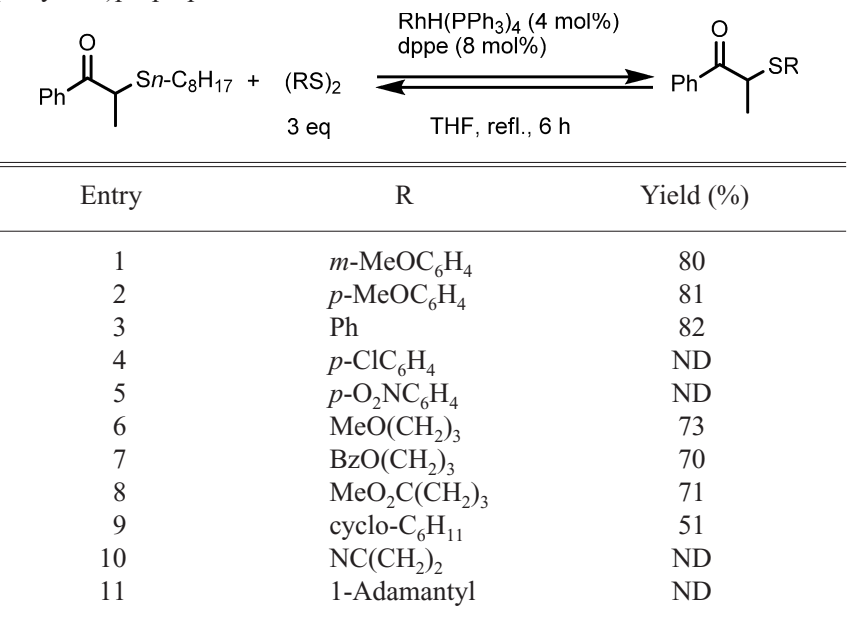

ND: not detected.

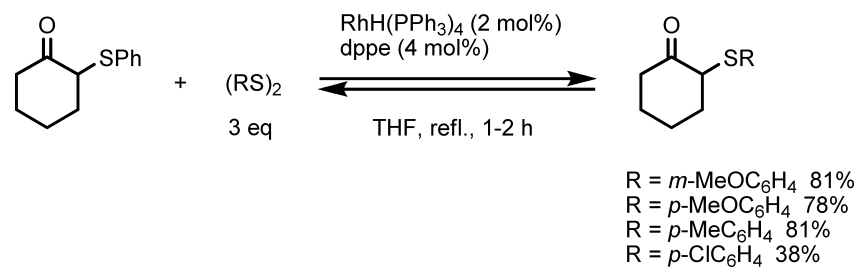

Chart 2

to determine the effect of the sulfur substituent (Table 2). The exchange reaction proceeded smoothly with several diaryl disulfides, which was consistent with the equilibrating nature of this reaction (entries 1-3). Aromatic disulfides possessing electron-withdrawing $p$-substituents, however, did not undergo the reaction (entries 4,5 ). The result may be partly due to the weaker $\mathrm{C}-\mathrm{S}$ bond energy of the exchange products, which decreased the thermodynamic stability of the products against the substrates. Alternatively, the catalyst was deactivated by thiolate ligands possessing electron-withdrawing $p$-substituents. Dialkyl disulfides also yielded the exchange products, which was again consistent with the reversibility of the reaction (entries 6-9). The inertness of bis(2-cyanoethyl) disulfide might be related to the lower reactivity of aromatic disulfides with electron-withdrawing $p$-substituents (entry 10). Tertiary di(1-adamantyl) disulfide was again inert (entry 11). This reaction was considerably affected by the steric and electronic nature of the disulfide substituent.

The reactivity of $\alpha$-phenylthio dialkyl ketones differed from that of the $\alpha$-phenylthio aryl ketones. 2-(Phenylthio)cyclohexanone reacted smoothly with aromatic disulfides (Chart 2). Bis( $p$-chlorophenyl) disulfide provided a lower yield of the product, which was consistent with the reactivity of $\alpha$-(octylthio)propiophenone noted earlier (Table 2, entries $4,5)$. The acyclic ketone 5-phenylthio-6-undecanone also reacted with a diaryl disulfide (Chart 3 ). In contrast, the reaction of these $\alpha$-phenylthio dialkyl ketones with bis(3methoxypropyl) disulfide resulted in yields less than $10 \%$ of the exchange products. The different reactivities of diaryl and dialkyl disulfides may be ascribed to the stronger S-S bond in the latter: organorhodium intermediates might have

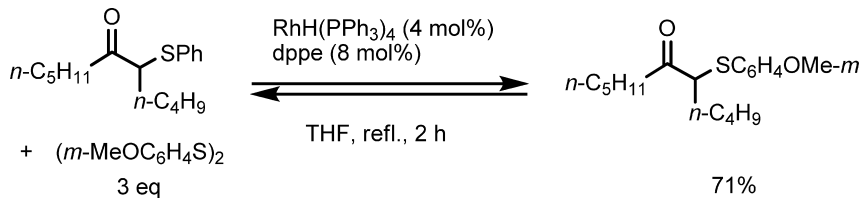

Chart 3
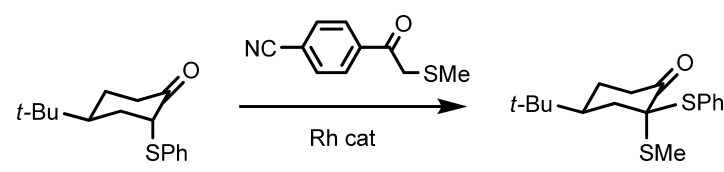

Chart 4

been formed by the oxidative addition of $\alpha$-phenylthio ketones, and the intermediates were sufficiently reactive with diaryl disulfides but not with dialkyl disulfides.

We previously reported the methylthio transfer reaction from $p$-cyano- $\alpha$-(methylthio)acetophenone to $\alpha$-phenylthio dialkyl ketones giving $\alpha$-methylthio- $\alpha$-phenylthio ketones, in which no phenylthio transfer was observed (Chart 4). ${ }^{14)}$ The selectivity may be related to the reactivity of this organothio exchange reaction, in which $\alpha$-phenylthio dialkyl ketones did not undergo the exchange reaction with dialkyl disulfides.

We have developed a novel method of synthesizing $\alpha$-keto sulfides. Note that the reaction is likely to involve the oxidative addition of $\alpha$-organothioketones to a low-valency rhodium complex to form novel organorhodium intermediates, and that the reactivity of such organometallic compounds may be of interest.

\section{Experimental}

${ }^{1} \mathrm{H}$ - and ${ }^{13} \mathrm{C}$-NMR spectra were recorded on Varian Mercury $(400 \mathrm{MHz})$ and JEOL JNM-ECA600 (600 MHz) NMR spectrometers. IR spectra were recorded on a JASCO FT/IR-410 spectrometer. Melting points were determined with a Yanagimoto micro-melting point apparatus without correction. High- and low-resolution mass spectra were measured on JEOL JMS-DX303, JEOL JMS-700, or JEOL JMS-T100GC mass spectrometer.

$\alpha$-Organothioketones were prepared by the treatment of ketones with lithium diisopropylamide (LDA) followed by diorganic disulfides in THF (or THF-hexamethylphosphoramide (HMPA)) at $-78^{\circ} \mathrm{C}$ to room temperature.

$\alpha$-(Phenylthio)acetophenone ${ }^{15)} \quad \operatorname{mp~} 52.0-52.5^{\circ} \mathrm{C}$ (hexane). Lit. $51-$ $52{ }^{\circ} \mathrm{C} .{ }^{1} \mathrm{H}-\mathrm{NMR}\left(400 \mathrm{MHz}, \mathrm{CDCl}_{3}\right) \delta: 4.28(2 \mathrm{H}, \mathrm{s}), 7.22(1 \mathrm{H}, \mathrm{t}, J=7.2 \mathrm{~Hz})$, $7.28(2 \mathrm{H}, \mathrm{t}, J=7.2 \mathrm{~Hz}), 7.39(2 \mathrm{H}, \mathrm{d}, J=7.2 \mathrm{~Hz}), 7.46(2 \mathrm{H}, \mathrm{t}, J=7.2 \mathrm{~Hz}), 7.58$ $(1 \mathrm{H}, \mathrm{t}, J=7.2 \mathrm{~Hz}), 7.94(2 \mathrm{H}, \mathrm{d}, J=7.2 \mathrm{~Hz}) \cdot{ }^{13} \mathrm{C}-\mathrm{NMR}\left(100 \mathrm{MHz}, \mathrm{CDCl}_{3}\right) \delta$ : $41.2,127.1,128.7,128.7,129.0,130.5,133.5,134.7,135.4,194.0$. IR (KBr): $3073,1671,1278 \mathrm{~cm}^{-1}$. MS (electron ionization (EI)) $\mathrm{m} / \mathrm{z}: 228\left(\mathrm{M}^{+}\right.$, $66 \%), 105\left(\mathrm{M}^{+}-\mathrm{C}_{7} \mathrm{H}_{7} \mathrm{~S}, 100 \%\right)$. High resolution (HR)-MS Calcd for $\mathrm{C}_{14} \mathrm{H}_{12} \mathrm{OS}: 228.0609$. Found: 228.0597.

$\alpha$-(Phenylthio)propiophenone ${ }^{16)}{ }^{1} \mathrm{H}-\mathrm{NMR}\left(400 \mathrm{MHz}, \mathrm{CDCl}_{3}\right) \delta: 1.53$ $(3 \mathrm{H}, \mathrm{d}, J=6.8 \mathrm{~Hz}), 4.63(1 \mathrm{H}, \mathrm{q}, J=6.8 \mathrm{~Hz}), 7.24-7.29(3 \mathrm{H}, \mathrm{m}), 7.34(2 \mathrm{H}$, dd, $J=8.0,2.0 \mathrm{~Hz}), 7.43(2 \mathrm{H}, \mathrm{t}, J=8.0 \mathrm{~Hz}), 7.54(1 \mathrm{H}, \mathrm{t}, J=8.0 \mathrm{~Hz}), 7.94(2 \mathrm{H}$, d, $J=7.2 \mathrm{~Hz}) .{ }^{13} \mathrm{C}-\mathrm{NMR}\left(100 \mathrm{MHz}, \mathrm{CDCl}_{3}\right) \delta: 16.9,46.1,128.5,128.5$, 128.6, 128.8, 131.6, 133.0, 134.5, 135.6, 196.2. IR (neat): 3059, 1681, 1447, 1233, $689 \mathrm{~cm}^{-1} \mathrm{MS}$ (EI) m/z: $242\left(\mathrm{M}^{+}, 37 \%\right), 137\left(\mathrm{M}^{+}-\mathrm{C}_{7} \mathrm{H}_{5} \mathrm{O}, 100 \%\right)$. HR-MS Calcd for $\mathrm{C}_{15} \mathrm{H}_{14} \mathrm{OS}$ : 242.0765 Found: 242.0769 .

$\boldsymbol{\alpha}$-(Phenylthio)butyrophenone ${ }^{17)} \quad \operatorname{mp} 38.0-39.0^{\circ} \mathrm{C}$ (hexane). ${ }^{1} \mathrm{H}-\mathrm{NMR}$ $\left(400 \mathrm{MHz}, \mathrm{CDCl}_{3}\right) \delta: 1.06(3 \mathrm{H}, \mathrm{t}, J=7.6 \mathrm{~Hz}), 1.87(1 \mathrm{H}$, ddq, $J=14.4,7.2$, $7.2 \mathrm{~Hz}), 2.02(1 \mathrm{H}$, ddq, $J=14.4,7.2,7.2 \mathrm{~Hz}), 4.38(1 \mathrm{H}, \mathrm{t}, J=7.6 \mathrm{~Hz}), 7.24$ $7.30(3 \mathrm{H}, \mathrm{m}), 7.34(2 \mathrm{H}, \mathrm{d}, J=8.0 \mathrm{~Hz}), 7.44(2 \mathrm{H}, \mathrm{t}, J=7.2 \mathrm{~Hz}), 7.56(1 \mathrm{H}, \mathrm{t}$, $J=8.0 \mathrm{~Hz}), 7.93(2 \mathrm{H}, \mathrm{d}, J=7.6 \mathrm{~Hz}) .{ }^{13} \mathrm{C}-\mathrm{NMR}\left(100 \mathrm{MHz}, \mathrm{CDCl}_{3}\right) \delta: 11.9$, $24.2,53.2,128.50,128.51,128.54,128.9,132.0,133.0,134.5,136.2,195.9$. IR (KBr): 3059, 2968, 1679, $1262 \mathrm{~cm}^{-1}$. MS (EI) $m / z: 256\left(\mathrm{M}^{+}, 29 \%\right), 151$ $\left(\mathrm{M}^{+}-\mathrm{C}_{7} \mathrm{H}_{5} \mathrm{O}, 100 \%\right)$. HR-MS Calcd for $\mathrm{C}_{16} \mathrm{H}_{16} \mathrm{OS}$ : 256.0922. Found: 256.0930 .

5-(Phenylthio)-6-undecanone ${ }^{1} \mathrm{H}-\mathrm{NMR}\left(400 \mathrm{MHz}, \mathrm{CDCl}_{3}\right) \quad \delta: 0.85$ $0.91(6 \mathrm{H}, \mathrm{m}), 1.18-1.37(7 \mathrm{H}, \mathrm{m}), 1.39-1.48(1 \mathrm{H}, \mathrm{m}), 1.50(2 \mathrm{H}$, quint, $J=$ 
$7.2 \mathrm{~Hz}), 1.63-1.73(1 \mathrm{H}, \mathrm{m}), 1.77-1.87(1 \mathrm{H}, \mathrm{m}), 2.56(2 \mathrm{H}, \mathrm{t}, J=7.2 \mathrm{~Hz})$ $3.62(1 \mathrm{H}, \mathrm{t}, J=7.2 \mathrm{~Hz}), 7.23-7.31(3 \mathrm{H}, \mathrm{m}), 7.35(2 \mathrm{H}, \mathrm{dd}, J=8.0,1.6 \mathrm{~Hz})$ ${ }^{13} \mathrm{C}-\mathrm{NMR}\left(100 \mathrm{MHz}, \mathrm{CDCl}_{3}\right) \delta: 13.8,13.9,22.4,23.5,29.4,30.1,31.3$, 39.2, 56.9, 127.7, 129.0, 132.3, 133.3, 207.6. IR (neat): 2956, 1709, 744 $691 \mathrm{~cm}^{-1}$. MS (EI) $\mathrm{m} / z: 278\left(\mathrm{M}^{+}, 25 \%\right), 179\left(\mathrm{M}^{+}-\mathrm{C}_{6} \mathrm{H}_{11} \mathrm{O}, 100 \%\right)$. HR-MS Calcd for $\mathrm{C}_{17} \mathrm{H}_{26} \mathrm{OS}: 278.1704$. Found: 278.1711

2-(Phenylthio)cyclohexanone ${ }^{18)} \quad{ }^{1} \mathrm{H}-\mathrm{NMR}\left(400 \mathrm{MHz}, \mathrm{CDCl}_{3}\right) \delta$ : 1.64 $1.72(1 \mathrm{H}, \mathrm{m}), 1.78-1.86(1 \mathrm{H}, \mathrm{m}), 1.88-1.98(2 \mathrm{H}, \mathrm{m}), 2.03-2.10(1 \mathrm{H}, \mathrm{m})$ $2.19-2.24(1 \mathrm{H}, \mathrm{m}), 2.26-2.32(1 \mathrm{H}, \mathrm{m}), 2.90(1 \mathrm{H}, \mathrm{ddd}, J=14.0,9.2,6.0$ $\mathrm{Hz}), 3.83(1 \mathrm{H}, \mathrm{t}, J=6.0 \mathrm{~Hz}), 7.23(1 \mathrm{H}, \mathrm{t}, J=7.6 \mathrm{~Hz}), 7.28(2 \mathrm{H}, \mathrm{t}, J=8.0 \mathrm{~Hz})$ $7.40(2 \mathrm{H}, \mathrm{d}, J=8.0 \mathrm{~Hz}) \cdot{ }^{13} \mathrm{C}-\mathrm{NMR}\left(100 \mathrm{MHz}, \mathrm{CDCl}_{3}\right) \delta: 22.6,27.2,33.9$, $39.0,56.2,127.3,128.9,131.8,133.7,207.6$. IR (neat): 3060, 2939, 2860 , 1708, 1482, 1438, 1123, 1023, 744, $691 \mathrm{~cm}^{-1}$. MS (EI) $\mathrm{m} / \mathrm{z}: 206\left(\mathrm{M}^{+}, 90 \%\right)$, $110\left(\mathrm{M}^{+}-\mathrm{C}_{6} \mathrm{H}_{8} \mathrm{O}, 100 \%\right)$. HR-MS Calcd for $\mathrm{C}_{12} \mathrm{H}_{14} \mathrm{OS}: 206.0765$. Found: 206.0765 .

$\boldsymbol{p}$-Methoxyphenyl $\boldsymbol{\alpha}$-(Phenylthio)methyl Ketone $^{19)}$ mp $\quad 87-88^{\circ} \mathrm{C}$ (hexane). Lit. $85-85.5^{\circ} \mathrm{C} .{ }^{1} \mathrm{H}-\mathrm{NMR}\left(400 \mathrm{MHz}, \mathrm{CDCl}_{3}\right) \delta: 3.88(3 \mathrm{H}, \mathrm{s})$ $4.24(2 \mathrm{H}, \mathrm{s}), 6.93(2 \mathrm{H}, \mathrm{ddd}, J=9.2,2.8,2.0 \mathrm{~Hz}), 7.22(1 \mathrm{H}, \mathrm{t}, J=7.6 \mathrm{~Hz}), 7.28$ $(2 \mathrm{H}, \mathrm{t}, J=6.8 \mathrm{~Hz}), 7.39(2 \mathrm{H}, \mathrm{d}, J=7.2 \mathrm{~Hz}), 7.93(2 \mathrm{H}, \mathrm{ddd}, J=8.8,2.8,2.0$ Hz). ${ }^{13} \mathrm{C}-\mathrm{NMR}\left(100 \mathrm{MHz}, \mathrm{CDCl}_{3}\right) \delta: 41.0,55.5,113.9,127.0,128.4,129.0$ 130.4, 131.1, 135.1, 163.8, 192.7. IR (KBr): 3060, 2925, 2846, 1670, 1599 , $1575,1509,1260,1172,1024,831,740,690 \mathrm{~cm}^{-1}$. MS (EI) $\mathrm{m} / z: 258\left(\mathrm{M}^{+}\right.$ $7 \%), 135\left(\mathrm{M}^{+}-\mathrm{C}_{7} \mathrm{H}_{7} \mathrm{~S}, 100 \%\right)$. HR-MS Calcd for $\mathrm{C}_{15} \mathrm{H}_{14} \mathrm{O}_{2} \mathrm{~S}: 258.0714$. Found: 258.0700 .

$\boldsymbol{\alpha}$-(Octylthio)propiophenone ${ }^{1} \mathrm{H}-\mathrm{NMR}\left(400 \mathrm{MHz}, \mathrm{CDCl}_{3}\right) \delta: 0.86(3 \mathrm{H}$, $\mathrm{t}, J=7.2 \mathrm{~Hz}), 1.21-1.30(10 \mathrm{H}, \mathrm{m}), 1.48(2 \mathrm{H}$, quint, $J=7.2 \mathrm{~Hz}), 1.56(3 \mathrm{H}, \mathrm{d}$ $J=6.4 \mathrm{~Hz}), 2.37(1 \mathrm{H}, \mathrm{dt}, J=11.6,7.2 \mathrm{~Hz}), 2.53(1 \mathrm{H}, \mathrm{dt}, J=12.4,7.2 \mathrm{~Hz})$ $4.32(1 \mathrm{H}, \mathrm{q}, J=6.4 \mathrm{~Hz}), 7.46(2 \mathrm{H}, \mathrm{td}, J=7.2,1.6 \mathrm{~Hz}), 7.56(1 \mathrm{H}, \mathrm{tt}, J=7.2$, $1.6 \mathrm{~Hz}), 8.01(2 \mathrm{H}, \mathrm{dd}, J=7.2,1.6 \mathrm{~Hz}) \cdot{ }^{13} \mathrm{C}-\mathrm{NMR}\left(100 \mathrm{MHz}, \mathrm{CDCl}_{3}\right) \delta: 14.1$, $16.4,22.6,28.7,28.9,29.1,29.1,29.2,31.7,41.5,128.5,128.5,132.9$ 135.7, 196.1. IR (neat): 2926, 1678, 716, $688 \mathrm{~cm}^{-1}$. MS (EI) $\mathrm{m} / z: 278\left(\mathrm{M}^{+}\right.$ 4\%), $173\left(\mathrm{M}^{+}-\mathrm{C}_{7} \mathrm{H}_{5} \mathrm{O}, 100 \%\right)$. HR-MS Calcd for $\mathrm{C}_{17} \mathrm{H}_{26} \mathrm{OS}: 278.1680$. Found: 278.1704 .

Typical Procedures for Organothio Exchange Reaction of Phenylthio Aryl Ketones, $\alpha-(m$-Methoxyphenylthio)acetophenone In a two-necked flask equipped with a reflux condenser were placed $\mathrm{RhH}\left(\mathrm{PPh}_{3}\right)_{4}(1 \mathrm{~mol} \%$, $2.9 \mathrm{mg})$, dppe $(2 \mathrm{~mol} \%, 2.0 \mathrm{mg})$, and $\alpha$-(phenylthio)acetophenone $(0.25$ $\mathrm{mmol}, 57 \mathrm{mg})$ under an argon atmosphere. After THF $(2 \mathrm{ml})$ and bis $(m-$ methoxyphenyl disulfide $(0.75 \mathrm{mmol}, 208 \mathrm{mg})$ were added, the solution was heated at reflux for $1 \mathrm{~h}$. The solvent was removed under reduced pressure, and flash chromatography (hexane/ethyl acetate $=15 / 1$ ) over silica gel gave $\alpha$-(m-methoxyphenylthio)acetophenone (53.9 mg, 84\%). ${ }^{1} \mathrm{H}-\mathrm{NMR} \quad(400$ $\left.\mathrm{MHz}, \mathrm{CDCl}_{3}\right) \delta: 3.76(3 \mathrm{H}, \mathrm{s}), 4.29(2 \mathrm{H}, \mathrm{s}), 6.75(1 \mathrm{H}, \mathrm{dd}, J=8.0,2.0 \mathrm{~Hz})$ $6.92(1 \mathrm{H}, \mathrm{t}, J=2.4 \mathrm{~Hz}), 6.95(1 \mathrm{H}, \mathrm{d}, J=8.0 \mathrm{~Hz}), 7.18(1 \mathrm{H}, \mathrm{t}, J=8.0 \mathrm{~Hz}), 7.45$ $(2 \mathrm{H}, \mathrm{t}, J=7.6 \mathrm{~Hz}), 7.57(1 \mathrm{H}, \mathrm{t}, J=8.0 \mathrm{~Hz}), 7.94(2 \mathrm{H}, \mathrm{d}, J=8.0 \mathrm{~Hz}) .{ }^{13} \mathrm{C}-\mathrm{NMR}$ $\left(100 \mathrm{MHz}, \mathrm{CDCl}_{3}\right) \delta: 41.1,55.3,112.8,115.3,122.2,128.5,128.5,129.7$ 133.3, 135.2, 135.9, 159.6, 193.8. IR (neat): 2937, 2834, 1681, 1590, 1277 , $1040 \mathrm{~cm}^{-1}$. MS (EI) $\mathrm{m} / \mathrm{z}: 258\left(\mathrm{M}^{+}, 65 \%\right), 105\left(\mathrm{M}^{+}-\mathrm{C}_{8} \mathrm{H}_{9} \mathrm{OS}, 100 \%\right)$. HRMS Calcd for $\mathrm{C}_{15} \mathrm{H}_{14} \mathrm{O}_{2} \mathrm{~S}: 258.0714$. Found: 258.0711.

$\alpha$-(p-Methylphenylthio)acetophenone ${ }^{20)}{ }^{1} \mathrm{H}-\mathrm{NMR}\left(400 \mathrm{MHz}, \mathrm{CDCl}_{3}\right)$ $\delta: 2.31(3 \mathrm{H}, \mathrm{s}), 4.20(2 \mathrm{H}, \mathrm{s}), 7.08(2 \mathrm{H}, \mathrm{d}, J=8.0 \mathrm{~Hz}), 7.28(2 \mathrm{H}, \mathrm{d}, J=8.0$ $\mathrm{Hz}), 7.45(2 \mathrm{H}, \mathrm{t}, J=7.6 \mathrm{~Hz}), 7.56(1 \mathrm{H}, \mathrm{t}, J=7.2 \mathrm{~Hz}), 7.92(2 \mathrm{H}, \mathrm{d}, J=7.6 \mathrm{~Hz})$ ${ }^{13} \mathrm{C}-\mathrm{NMR}\left(100 \mathrm{MHz}, \mathrm{CDCl}_{3}\right) \delta: 21.2,41.9,128.5,128.6,129.7,130.7$, 131.4, 133.2, 137.4, 141.4, 193.9. IR (neat): $2938,2835,1680,1277 \mathrm{~cm}^{-1}$ MS (EI) $m / z: 242\left(\mathrm{M}^{+}, 65 \%\right), 105\left(\mathrm{M}^{+}-\mathrm{C}_{8} \mathrm{H}_{9} \mathrm{~S}, 100 \%\right)$. HR-MS Calcd for $\mathrm{C}_{15} \mathrm{H}_{14} \mathrm{OS}: 242.0765$. Found: 242.0756 .

$\boldsymbol{\alpha}$-(3-Methoxypropylthio)acetophenone ${ }^{1} \mathrm{H}-\mathrm{NMR} \quad\left(400 \mathrm{MHz}, \mathrm{CDCl}_{3}\right)$ $\delta: 1.87(2 \mathrm{H}$, quint, $J=7.2 \mathrm{~Hz}), 2.65(2 \mathrm{H}, \mathrm{t}, J=7.2 \mathrm{~Hz}), 3.31(3 \mathrm{H}, \mathrm{s}), 3.43$ $(2 \mathrm{H}, \mathrm{t}, J=6.8 \mathrm{~Hz}), 3.80(2 \mathrm{H}, \mathrm{s}), 7.46(2 \mathrm{H}, \mathrm{t}, J=7.2 \mathrm{~Hz}), 7.57(1 \mathrm{H}, \mathrm{t}, J=7.2$ $\mathrm{Hz}), 7.97(2 \mathrm{H}, \mathrm{d}, J=7.2 \mathrm{~Hz}) .{ }^{13} \mathrm{C}-\mathrm{NMR}\left(100 \mathrm{MHz}, \mathrm{CDCl}_{3}\right) \delta: 29.1,29.2$ $37.2,58.6,70.9,128.5,128.6,133.2,135.1,194.2$. IR (neat): 3060, 2926 , $1673,1277,1116 \mathrm{~cm}^{-1}$. MS (EI) $\mathrm{m} / z: 224\left(\mathrm{M}^{+}, 42 \%\right), 105\left(\mathrm{M}^{+}-\mathrm{C}_{5} \mathrm{H}_{11} \mathrm{OS}\right.$, $100 \%)$. HR-MS Calcd for $\mathrm{C}_{12} \mathrm{H}_{16} \mathrm{O}_{2} \mathrm{~S}: 224.0871$. Found: 224.0879 .

p-Methoxyphenyl $\boldsymbol{\alpha}$-(m-Methoxyphenylthio)methyl Ketone ${ }^{1} \mathrm{H}-\mathrm{NMR}$ $\left(400 \mathrm{MHz}, \mathrm{CDCl}_{3}\right) \delta: 3.76(3 \mathrm{H}, \mathrm{s}), 3.87(3 \mathrm{H}, \mathrm{s}), 4.25(2 \mathrm{H}, \mathrm{s}), 6.74(1 \mathrm{H}, \mathrm{dd}$, $J=8.0,2.0 \mathrm{~Hz}), 6.91-6.96(4 \mathrm{H}, \mathrm{m}), 7.18(1 \mathrm{H}, \mathrm{t}, J=8.0 \mathrm{~Hz}), 7.92(2 \mathrm{H}, \mathrm{d}, J=$ $8.8 \mathrm{~Hz}) .{ }^{13} \mathrm{C}-\mathrm{NMR}\left(100 \mathrm{MHz}, \mathrm{CDCl}_{3}\right) \delta: 40.8,55.3,55.5,112.6,113.7$, $115.1,121.9,128.2,129.7,130.9,136.2,159.6,163.6,192.4$. IR (neat) 2936, 2837, 1670,1597, 1479, 1260,1173,1036 $\mathrm{cm}^{-1}$. MS (EI) $\mathrm{m} / \mathrm{z}: 288$ $\left(\mathrm{M}^{+}, 27 \%\right), 135\left(\mathrm{M}^{+}-\mathrm{C}_{8} \mathrm{H}_{9} \mathrm{OS}, 100 \%\right)$. HR-MS Calcd for $\mathrm{C}_{16} \mathrm{H}_{16} \mathrm{O}_{3} \mathrm{~S}$ 288.0820. Found: 288.0826 .

$\boldsymbol{\alpha}$-(m-Methoxyphenylthio)propiophenone ${ }^{1} \mathrm{H}-\mathrm{NMR} \quad\left(400 \mathrm{MHz}, \mathrm{CDCl}_{3}\right)$ $\delta: 1.56(3 \mathrm{H}, \mathrm{d}, J=6.4 \mathrm{~Hz}), 3.74(3 \mathrm{H}, \mathrm{s}), 4.65(1 \mathrm{H}, \mathrm{q}, J=6.8 \mathrm{~Hz}), 6.83(1 \mathrm{H}$, dd, $J=8.0,2.4 \mathrm{~Hz}), 6.86(1 \mathrm{H}, \mathrm{s}), 6.93(1 \mathrm{H}, \mathrm{d}, J=7.2 \mathrm{~Hz}), 7.18(1 \mathrm{H}, \mathrm{t}, J=$
$8.0 \mathrm{~Hz}), 7.44(2 \mathrm{H}, \mathrm{t}, J=7.2 \mathrm{~Hz}), 7.55(1 \mathrm{H}, \mathrm{t}, J=7.2 \mathrm{~Hz}), 7.94(2 \mathrm{H}, \mathrm{d}, J=$ $6.4 \mathrm{~Hz}) .{ }^{13} \mathrm{C}-\mathrm{NMR}\left(100 \mathrm{MHz}, \mathrm{CDCl}_{3}\right) \delta: 17.3,46.4,55.3,114.4,119.0$ $126.1,128.4,128.5,129.6,133.0,133.1,135.5,159.4,196.2$. IR (neat): 2930, 1681, 1589, 1479, 1231, $1040 \mathrm{~cm}^{-1}$. MS (EI) $\mathrm{m} / \mathrm{z}: 272\left(\mathrm{M}^{+}, 47 \%\right)$ $167\left(\mathrm{M}^{+}-\mathrm{C}_{7} \mathrm{H}_{5} \mathrm{O}, 100 \%\right)$. HR-MS Calcd for $\mathrm{C}_{16} \mathrm{H}_{16} \mathrm{O}_{2} \mathrm{~S}: 272.0871$. Found: 272.0862 .

$\boldsymbol{\alpha}$-(m-Methoxyphenylthio)ethyl $\boldsymbol{p}$-Methylphenyl Ketone ${ }^{1} \mathrm{H}$-NMR (400 $\left.\mathrm{MHz}, \mathrm{CDCl}_{3}\right) \delta: 1.54(3 \mathrm{H}, \mathrm{d}, J=6.8 \mathrm{~Hz}), 2.41(3 \mathrm{H}, \mathrm{s}), 3.74(3 \mathrm{H}, \mathrm{s}), 4.64$ $(1 \mathrm{H}, \mathrm{q}, J=6.8 \mathrm{~Hz}), 6.82(1 \mathrm{H}, \mathrm{dd}, J=8.0,2.0 \mathrm{~Hz}), 6.86(1 \mathrm{H}, \mathrm{d}, J=1.6 \mathrm{~Hz})$ $6.94(1 \mathrm{H}, \mathrm{d}, J=8.0 \mathrm{~Hz}), 7.17(1 \mathrm{H}, \mathrm{t}, J=7.6 \mathrm{~Hz}), 7.23(2 \mathrm{H}, \mathrm{d}, J=8.0 \mathrm{~Hz}), 7.84$ $(2 \mathrm{H}, \mathrm{d}, J=8.8 \mathrm{~Hz}) .{ }^{13} \mathrm{C}-\mathrm{NMR}\left(100 \mathrm{MHz}, \mathrm{CDCl}_{3}\right) \delta: 17.4,21.8,46.4,55.3$, $114.3,118.8,125.9,128.6,129.1,129.5,132.9,141.2,143.8,159.4,195.9$. IR (neat): 2929, 1675, 1589, 1478, 1245, 1040, $949 \mathrm{~cm}^{-1}$. MS (EI) $\mathrm{m} / \mathrm{z}: 286$ $\left(\mathrm{M}^{+}, 77 \%\right), 119\left(\mathrm{M}^{+}-\mathrm{C}_{9} \mathrm{H}_{11} \mathrm{OS}, 100 \%\right)$. HR-MS Calcd for $\mathrm{C}_{17} \mathrm{H}_{18} \mathrm{SO}_{2}$ : 286.1028. Found: 286.1018

$\boldsymbol{\alpha}$-(m-Methoxyphenylthio)butyrophenone ${ }^{1} \mathrm{H}-\mathrm{NMR} \quad\left(400 \mathrm{MHz}, \mathrm{CDCl}_{3}\right)$ $\delta: 1.06(3 \mathrm{H}, \mathrm{t}, J=7.2 \mathrm{~Hz}), 1.89(1 \mathrm{H}, \mathrm{ddq}, J=14.4,7.2,7.2 \mathrm{~Hz}), 2.07(1 \mathrm{H}$, ddq, $J=14.4,7.2,7.2 \mathrm{~Hz}), 3.72(3 \mathrm{H}, \mathrm{s}), 4.40(1 \mathrm{H}, \mathrm{t}, J=7.2 \mathrm{~Hz}), 6.81(1 \mathrm{H}, \mathrm{dd}$ $J=8.4,2.4 \mathrm{~Hz}), 6.84(1 \mathrm{H}, \mathrm{bs}), 6.92(1 \mathrm{H}, \mathrm{d}, J=7.6 \mathrm{~Hz}), 7.17(1 \mathrm{H}, \mathrm{t}, J=7.6$ $\mathrm{Hz}), 7.42(2 \mathrm{H}, \mathrm{t}, J=7.6 \mathrm{~Hz}), 7.54(1 \mathrm{H}, \mathrm{t}, J=7.2 \mathrm{~Hz}), 7.91(2 \mathrm{H}, \mathrm{d}, J=7.2 \mathrm{~Hz})$ ${ }^{13} \mathrm{C}-\mathrm{NMR}\left(100 \mathrm{MHz}, \mathrm{CDCl}_{3}\right) \delta: 12.1,24.5,53.4,55.3,114.3,118.9,126.0$, $128.4,128.4,129.5,132.9,133.4,136.1,159.3,195.9$. IR (neat): 2965 , $1680,1589,1477,1247,1040 \mathrm{~cm}^{-1}$. MS (EI) $m / z: 286\left(\mathrm{M}^{+}, 47 \%\right), 181$ $\left(\mathrm{M}^{+}-\mathrm{C}_{7} \mathrm{H}_{5} \mathrm{O}, 100 \%\right)$. HR-MS Calcd for $\mathrm{C}_{17} \mathrm{H}_{18} \mathrm{O}_{2} \mathrm{~S}$ : 286.1028. Found: 286.1018.

$\boldsymbol{\alpha}$-(3-Methoxypropylthio)butyrophenone ${ }^{1} \mathrm{H}-\mathrm{NMR}\left(400 \mathrm{MHz}, \mathrm{CDCl}_{3}\right)$ $\delta: 1.03(3 \mathrm{H}, \mathrm{t}, J=7.2 \mathrm{~Hz}), 1.74(2 \mathrm{H}$, quint, $J=6.4 \mathrm{~Hz}), 1.86(1 \mathrm{H}, \mathrm{ddq}, J=$ $14.0,7.2,7.2 \mathrm{~Hz}), 2.10(1 \mathrm{H}, \mathrm{ddq}, J=14.0,7.2,7.2 \mathrm{~Hz}), 2.45(1 \mathrm{H}, \mathrm{dt}, J=$ $14.0,7.2 \mathrm{~Hz}), 2.58(1 \mathrm{H}, \mathrm{dt}, J=14.0,7.2 \mathrm{~Hz}), 3.27(3 \mathrm{H}, \mathrm{s}), 3.35(2 \mathrm{H}, \mathrm{td}, J=$ $6.0,2.0 \mathrm{~Hz}), 4.05(1 \mathrm{H}, \mathrm{t}, J=7.2 \mathrm{~Hz}), 7.45(2 \mathrm{H}, \mathrm{t}, J=7.6 \mathrm{~Hz}), 7.55(1 \mathrm{H}, \mathrm{t}, J=$ $7.2 \mathrm{~Hz}), 7.98(2 \mathrm{H}$, dd, $J=8.0,1.2 \mathrm{~Hz}) \cdot{ }^{13} \mathrm{C}-\mathrm{NMR}\left(100 \mathrm{MHz}, \mathrm{CDCl}_{3}\right) \delta: 12.2$, $23.6,25.8,29.5,48.8,58.6,71.2,128.3,129.4,132.8,136.0,195.5$. IR (neat): 2929, 2873, 1675, 1447, 1223, $1118 \mathrm{~cm}^{-1}$. MS (EI) m/z: $252\left(\mathrm{M}^{+}\right.$ $26 \%), 89\left(\mathrm{M}^{+}-\mathrm{C}_{10} \mathrm{H}_{11} \mathrm{~S}, 100 \%\right)$. HR-MS Calcd for $\mathrm{C}_{14} \mathrm{H}_{20} \mathrm{O}_{3} \mathrm{~S}: 252.1184$ Found: 252.1183 .

$\boldsymbol{\alpha}$-(Cyclopentylthio)butyrophenone ${ }^{1} \mathrm{H}-\mathrm{NMR} \quad\left(400 \mathrm{MHz}, \mathrm{CDCl}_{3}\right) \delta$ : $1.02(3 \mathrm{H}, \mathrm{t}, J=7.2 \mathrm{~Hz}), 1.32-1.54(4 \mathrm{H}, \mathrm{m}), 1.59-1.70(2 \mathrm{H}, \mathrm{m}), 1.84$ $1.92(2 \mathrm{H}, \mathrm{m}), 1.94-2.04(1 \mathrm{H}, \mathrm{m}), 2.13(1 \mathrm{H}, \mathrm{ddq}, J=15.2,7.6,7.6 \mathrm{~Hz}), 3.02$ $(1 \mathrm{H}$, quint, $J=7.6 \mathrm{~Hz}), 4.08(1 \mathrm{H}, \mathrm{t}, J=7.6 \mathrm{~Hz}), 7.46(2 \mathrm{H}, \mathrm{t}, J=7.6 \mathrm{~Hz}), 7.55$ $(1 \mathrm{H}, \mathrm{t}, J=7.6 \mathrm{~Hz}), 7.99(2 \mathrm{H}, \mathrm{d}, J=7.6 \mathrm{~Hz}) .{ }^{13} \mathrm{C}-\mathrm{NMR}\left(100 \mathrm{MHz}, \mathrm{CDCl}_{3}\right) \delta$ $12.4,24.8,24.9,25.0,34.3,35.1,42.4,50.0,128.4,128.4,132.8,136.3$, 196.8. IR (neat): $2962,2871,1677,1447,1262,1224 \mathrm{~cm}^{-1}$. MS (EI) $\mathrm{m} / \mathrm{z}$ : $248\left(\mathrm{M}^{+}, 4 \%\right), 143\left(\mathrm{M}^{+}-\mathrm{C}_{7} \mathrm{H}_{5} \mathrm{O}, 100 \%\right)$. HR-MS Calcd for $\mathrm{C}_{15} \mathrm{H}_{20} \mathrm{OS}$ : 248.1235. Found: 248.1232

$\boldsymbol{\alpha}$-(Cyclohexylthio)butyrophenone ${ }^{1} \mathrm{H}-\mathrm{NMR}\left(400 \mathrm{MHz}, \mathrm{CDCl}_{3}\right) \delta: 1.01$ $(3 \mathrm{H}, \mathrm{t}, J=7.6 \mathrm{~Hz}), 1.15-1.34(5 \mathrm{H}, \mathrm{m}), 1.52-1.55(1 \mathrm{H}, \mathrm{m}), 1.65-1.70$ $(2 \mathrm{H}, \mathrm{m}), 1.81-1.91(3 \mathrm{H}, \mathrm{m}), 2.12(1 \mathrm{H}, \mathrm{ddq}, J=15.2,7.6,7.6 \mathrm{~Hz}), 2.64$ $2.73(1 \mathrm{H}, \mathrm{m}), 4.07(1 \mathrm{H}, \mathrm{t}, J=7.6 \mathrm{~Hz}), 7.45(2 \mathrm{H}, \mathrm{t}, J=8.0 \mathrm{~Hz}), 7.55(1 \mathrm{H}, \mathrm{t}$ $J=8.0 \mathrm{~Hz}), 7.99(2 \mathrm{H}, \mathrm{d}, J=8.0 \mathrm{~Hz}){ }^{13} \mathrm{C}-\mathrm{NMR}\left(100 \mathrm{MHz}, \mathrm{CDCl}_{3}\right) \delta: 12.4$, 25.1, 25.6, 34.4, 34.9, 42.7, 49.3, 128.36, 128.40, 132.7, 136.2, 196.9. IR (neat): 2930, 2852,1677,1578, 1262,1224, $999 \mathrm{~cm}^{-1}$. MS (EI) $\mathrm{m} / \mathrm{z}: 262$ $\left(\mathrm{M}^{+}, 6 \%\right), 157\left(\mathrm{M}^{+}-\mathrm{C}_{7} \mathrm{H}_{5} \mathrm{O}, 100 \%\right)$. HR-MS Calcd for $\mathrm{C}_{16} \mathrm{H}_{22} \mathrm{SO}$ : 262.1391. Found: 262.1373

Typical Procedures for Organothio Exchange Reaction of Alkylthio Ketone, $\alpha$-( $m$-Methoxyphenylthio)propiophenone In a two-necked flask equipped with a reflux condenser were place $\alpha$-(octylthio)propiophenone (139.2 $\mathrm{mg}, 0.5 \mathrm{mmol})$, bis ( $m$-methoxyphenyl) disulfide $(315.5 \mathrm{mg}, 1.5 \mathrm{mmol})$, $\mathrm{RhH}\left(\mathrm{PPh}_{3}\right)_{4}(11.6 \mathrm{mg}, 2.0 \mathrm{~mol} \%)$ and dppe $(8.0 \mathrm{mg}, 4.0 \mathrm{~mol} \%)$ in THF $(2 \mathrm{ml})$ under an argon atmosphere, and the solution was heated at reflux for $3 \mathrm{~h}$. The solvent was removed under reduced pressure, and the residue was purified by flash column chromatography on silica gel giving $\alpha$ - $(m-$ methoxyphenylthio)propiophenone (109.2 $\mathrm{mg}, 80 \%)$, and $m$-methoxyphenyl octyl disulfide (110.4 mg, 78\%).

$\boldsymbol{\alpha}$-(p-Methoxyphenylthio)propiophenone $\mathrm{mp} 85-87^{\circ} \mathrm{C}$ (hexane). ${ }^{1} \mathrm{H}-$ NMR $\left(400 \mathrm{MHz}, \mathrm{CDCl}_{3}\right) \delta: 1.46(3 \mathrm{H}, \mathrm{d}, J=7.2 \mathrm{~Hz}), 3.76(3 \mathrm{H}, \mathrm{s}), 4.49(1 \mathrm{H}$, q, $J=6.8 \mathrm{~Hz}), 6.79(2 \mathrm{H}, \mathrm{d}, J=8.8 \mathrm{~Hz}), 7.25(2 \mathrm{H}, \mathrm{d}, J=8.8 \mathrm{~Hz}), 7.43(2 \mathrm{H}, \mathrm{t}$, $J=8.0 \mathrm{~Hz}), 7.54(1 \mathrm{H}, \mathrm{t}, J=8.0 \mathrm{~Hz}), 7.96(2 \mathrm{H}, \mathrm{d}, J=7.2 \mathrm{~Hz}) .{ }^{13} \mathrm{C}-\mathrm{NMR}(100$ $\left.\mathrm{MHz}, \mathrm{CDCl}_{3}\right) \delta: 16.4,46.0,55.1,114.3,121.2,128.4,128.5,132.8,135.7$, 137.4, 160.2, 195.9. IR (KBr): 2966, 1677, 1593, 1494, 1249, 1030, 828, $708,688 \mathrm{~cm}^{-1}$. MS (EI) $\mathrm{m} / \mathrm{z}: 272\left(\mathrm{M}^{+}, 53 \%\right), 167\left(\mathrm{M}^{+}-\mathrm{C}_{7} \mathrm{H}_{5} \mathrm{O}, 100 \%\right)$. HR-MS Calcd for $\mathrm{C}_{16} \mathrm{H}_{16} \mathrm{O}_{2} \mathrm{~S}: 272.0853$. Found: 272.0871 .

$\alpha$-(3-Methyloxypropylthio)propiophenone ${ }^{1} \mathrm{H}-\mathrm{NMR}\left(400 \mathrm{MHz}, \mathrm{CDCl}_{3}\right)$ $\delta: 1.57(3 \mathrm{H}, \mathrm{d}, J=6.8 \mathrm{~Hz}), 1.75(2 \mathrm{H}, \mathrm{m}, J=7.6 \mathrm{~Hz}), 2.47(1 \mathrm{H}, \mathrm{dt}, J=12.4$, $7.2 \mathrm{~Hz}), 2.62(1 \mathrm{H}, \mathrm{dt}, J=12.4,7.2 \mathrm{~Hz}), 3.27(3 \mathrm{H}, \mathrm{s}), 3.36(2 \mathrm{H}, \mathrm{td}, J=6.8$, 
$2.0 \mathrm{~Hz}), 4.34(1 \mathrm{H}, \mathrm{q}, J=7.2 \mathrm{~Hz}), 7.47(2 \mathrm{H}, \mathrm{t}, J=7.6 \mathrm{~Hz}), 7.59(1 \mathrm{H}, \mathrm{t}, J=7.6$ $\mathrm{Hz}), 8.01(2 \mathrm{H}, \mathrm{d}, J=7.6 \mathrm{~Hz}) .{ }^{13} \mathrm{C}-\mathrm{NMR}\left(100 \mathrm{MHz}, \mathrm{CDCl}_{3}\right) \delta: 16.4,25.5$, $29.3,41.5,58.5,71.0,128.5,128.5,132.9,135.5,196.0$. IR (neat): 2927 , 1678, 1117, $717 \mathrm{~cm}^{-1}$. MS (EI) m/z: $238\left(\mathrm{M}^{+}, 30 \%\right), 105\left(\mathrm{M}^{+}-\mathrm{C}_{6} \mathrm{H}_{13} \mathrm{OS}\right.$ $100 \%$ ). HR-MS Calcd for $\mathrm{C}_{13} \mathrm{H}_{18} \mathrm{O}_{2} \mathrm{~S}: 238.1028$. Found: 238.1014 .

$\boldsymbol{\alpha}$-(3-Benzoyloxypropylthio)propiophenone ${ }^{1} \mathrm{H}-\mathrm{NMR}\left(400 \mathrm{MHz}, \mathrm{CDCl}_{3}\right)$ $\delta: 1.59(3 \mathrm{H}, \mathrm{d}, J=7.2 \mathrm{~Hz}), 1.97(2 \mathrm{H}$, quint, $J=6.8 \mathrm{~Hz}), 2.57(1 \mathrm{H}, \mathrm{dt}, J=13.2$, $7.2 \mathrm{~Hz}), 2.71(1 \mathrm{H}, \mathrm{dt}, J=13.2,7.2 \mathrm{~Hz}), 4.28-4.40(3 \mathrm{H}, \mathrm{m}), 7.44(2 \mathrm{H}, \mathrm{t}, J=$ $8.0 \mathrm{~Hz}), 7.45(2 \mathrm{H}, \mathrm{t}, J=8.0 \mathrm{~Hz}), 7.56(1 \mathrm{H}, \mathrm{t}, J=8.0 \mathrm{~Hz}), 7.56(1 \mathrm{H}, \mathrm{t}, J=8.0$ $\mathrm{Hz}), 8.01(2 \mathrm{H}, \mathrm{d}, J=6.8 \mathrm{~Hz}), 8.02(2 \mathrm{H}, \mathrm{d}, J=7.2 \mathrm{~Hz}) .{ }^{13} \mathrm{C}-\mathrm{NMR}(100 \mathrm{MHz}$ $\left.\mathrm{CDCl}_{3}\right) \delta: 16.4,25.2,28.6,41.4,63.3,128.3,128.5,128.6,129.5,130.1$, 132.9, 133.1, 135.4, 166.4, 196.0. IR (neat): 2929, 1719, 1676, 1274, 1115 $713,688 \mathrm{~cm}^{-1}$. MS (EI) $\mathrm{m} / z: 328\left(\mathrm{M}^{+}, 7 \%\right), 223\left(\mathrm{M}^{+}-\mathrm{C}_{7} \mathrm{H}_{5} \mathrm{O}, 100 \%\right)$. HRMS Calcd for $\mathrm{C}_{19} \mathrm{H}_{20} \mathrm{O}_{3} \mathrm{~S}: 328.1133$. Found: 328.1133 .

$\alpha$-(3-Methoxycarbonylpropylthio)propiophenone ${ }^{1} \mathrm{H}-\mathrm{NMR} \quad(400 \mathrm{MHz}$, $\left.\mathrm{CDCl}_{3}\right) \delta: 1.57(3 \mathrm{H}, \mathrm{d}, J=7.2 \mathrm{~Hz}), 1.83(1 \mathrm{H}$, quint, $J=7.2 \mathrm{~Hz}), 1.84(1 \mathrm{H}$ quint, $J=7.2 \mathrm{~Hz}), 2.35(2 \mathrm{H}, \mathrm{t}, J=7.2 \mathrm{~Hz}), 2.44(1 \mathrm{H}, \mathrm{dt}, J=12.4,7.2 \mathrm{~Hz})$ $2.59(1 \mathrm{H}, \mathrm{dt}, J=12.4,7.2 \mathrm{~Hz}), 3.65(3 \mathrm{H}, \mathrm{s}), 4.35(1 \mathrm{H}, \mathrm{q}, J=6.8 \mathrm{~Hz}), 7.47$ $(2 \mathrm{H}, \mathrm{t}, J=7.2 \mathrm{~Hz}), 7.57(1 \mathrm{H}, \mathrm{tt}, J=7.2,2.0 \mathrm{~Hz}), 8.00(2 \mathrm{H}, \mathrm{dd}, J=7.2,2.4$ $\mathrm{Hz}) .{ }^{13} \mathrm{C}-\mathrm{NMR}\left(100 \mathrm{MHz}, \mathrm{CDCl}_{3}\right) \delta: 16.5,24.5,28.0,32.8,41.2,51.6$ 128.5, 128.6, 133.1, 135.5, 173.3, 196.1. IR (neat): 2951, 1737, 1677, 1235, $719,688 \mathrm{~cm}^{-1}$. MS (EI) $\mathrm{m} / z$ : $266\left(\mathrm{M}^{+}, 30 \%\right), 161\left(\mathrm{M}^{+}-\mathrm{C}_{7} \mathrm{H}_{5} \mathrm{O}, 100 \%\right)$ HR-MS Calcd for $\mathrm{C}_{14} \mathrm{H}_{18} \mathrm{O}_{3} \mathrm{~S}: 266.0977$. Found: 266.0973.

$\boldsymbol{\alpha}$-(Cyclohexylthio)propiophenone ${ }^{1} \mathrm{H}-\mathrm{NMR} \quad\left(400 \mathrm{MHz}, \mathrm{CDCl}_{3}\right) \quad \delta$ $1.17-1.39(5 \mathrm{H}, \mathrm{m}), 1.53-1.59(1 \mathrm{H}, \mathrm{m}), 1.59(3 \mathrm{H}, \mathrm{d}, J=6.4 \mathrm{~Hz}), 1.63-1.72$ $(2 \mathrm{H}, \mathrm{m}), 1.80-1.84(1 \mathrm{H}, \mathrm{m}), 1.92-1.94(1 \mathrm{H}, \mathrm{m}), 2.71-2.77(1 \mathrm{H}, \mathrm{m}), 4.37$ $(1 \mathrm{H}, \mathrm{q}, J=6.8 \mathrm{~Hz}), 7.46(2 \mathrm{H}, \mathrm{t}, J=7.2 \mathrm{~Hz}), 7.56(1 \mathrm{H}, \mathrm{t}, J=7.2 \mathrm{~Hz}), 8.01(2 \mathrm{H}$, d, $J=7.2 \mathrm{~Hz}) \cdot{ }^{13} \mathrm{C}-\mathrm{NMR}\left(100 \mathrm{MHz}, \mathrm{CDCl}_{3}\right) \delta: 17.7,25.2,26.0,34.2,34.7$, 41.9, 42.5, 128.5, 128.6, 132.9, 135.8, 197.4. IR (neat): 2929, 1677, 1447, $712,688 \mathrm{~cm}^{-1}$. MS (EI) $\mathrm{m} / \mathrm{z}: 248\left(\mathrm{M}^{+}, 8 \%\right), 143\left(\mathrm{M}^{+}-\mathrm{C}_{7} \mathrm{H}_{5} \mathrm{O}, 100 \%\right)$. HRMS Calcd for $\mathrm{C}_{15} \mathrm{H}_{20} \mathrm{OS}: 248.1235$. Found: 248.1216 .

2-(m-Methoxyphenylthio)cyclohexanone ${ }^{21)}{ }^{1} \mathrm{H}-\mathrm{NMR}\left(400 \mathrm{MHz}, \mathrm{CDCl}_{3}\right)$ $\delta: 1.66-1.73(1 \mathrm{H}, \mathrm{m}), 1.82-1.98(3 \mathrm{H}, \mathrm{m}), 2.03-2.11(1 \mathrm{H}, \mathrm{m}), 2.21-$ $2.33(2 \mathrm{H}, \mathrm{m}), 2.87-2.94(1 \mathrm{H}, \mathrm{m}), 3.79(3 \mathrm{H} . \mathrm{s}), 3.86(1 \mathrm{H}, \mathrm{t}, J=6.6 \mathrm{~Hz}), 6.78$ $(1 \mathrm{H}, \mathrm{dd}, J=8.0,2.0 \mathrm{~Hz}), 6.95-6.98(2 \mathrm{H}, \mathrm{m}), 7.20(1 \mathrm{H}, \mathrm{t}, J=7.6 \mathrm{~Hz}) \cdot{ }^{13} \mathrm{C}-$ NMR $\left(100 \mathrm{MHz}, \mathrm{CDCl}_{3}\right) \delta: 22.7,27.3,34.0,39.1,55.2,56.2,113.1,116.7$, 123.6, 129.7, 135.1, 159.7, 207.7. IR (neat): 2939, 1709, 1590, 1479, 1230, $776,688 \mathrm{~cm}^{-1}$. MS (EI) m/z: $236\left(\mathrm{M}^{+}, 89 \%\right), 140\left(\mathrm{M}^{+}-\mathrm{C}_{6} \mathrm{H}_{8} \mathrm{O}, 100 \%\right)$. HR-MS Calcd for $\mathrm{C}_{13} \mathrm{H}_{16} \mathrm{O}_{2} \mathrm{~S}: 236.0871$. Found: 236.0852 .

2-(p-Methoxyphenylthio)cyclohexanone ${ }^{1} \mathrm{H}-\mathrm{NMR}\left(400 \mathrm{MHz}, \mathrm{CDCl}_{3}\right)$ $\delta: 1.61-1.69(1 \mathrm{H}, \mathrm{m}), 1.77-1.85(1 \mathrm{H}, \mathrm{m}), 1.88-1.97(2 \mathrm{H}, \mathrm{m}), 2.00-$ $2.08(1 \mathrm{H}, \mathrm{m}), 2.13-2.21(1 \mathrm{H}, \mathrm{m}), 2.23-2.29(1 \mathrm{H}, \mathrm{m}), 2.87-2.94(1 \mathrm{H}, \mathrm{m})$ $3.66(1 \mathrm{H}, \mathrm{t}, J=6.8 \mathrm{~Hz}), 3.78(3 \mathrm{H}, \mathrm{s}), 6.82(2 \mathrm{H}, \mathrm{d}, J=6.8 \mathrm{~Hz}), 7.36(2 \mathrm{H}, \mathrm{d}$, $J=6.8 \mathrm{~Hz}) .{ }^{13} \mathrm{C}-\mathrm{NMR}\left(100 \mathrm{MHz}, \mathrm{CDCl}_{3}\right) \delta: 22.3,27.1,33.5,38.8,55.1$, 57.6, 114.5, 123.6, 135.3, 159.7, 207.6. IR (neat): 2939, 1704, 1592, 1494 $1245,1122,1029,828 \mathrm{~cm}^{-1}$. MS (EI) $\mathrm{m} / \mathrm{z}: 236\left(\mathrm{M}^{+}, 100 \%\right), 140\left(\mathrm{M}^{+}\right.$ $\left.\mathrm{C}_{6} \mathrm{H}_{8} \mathrm{O}, 95 \%\right)$. HR-MS Calcd for $\mathrm{C}_{13} \mathrm{H}_{16} \mathrm{O}_{2} \mathrm{~S}: 236.0873$. Found: 236.0871

2-(p-Methylphenylthio)cyclohexanone ${ }^{1} \mathrm{H}-\mathrm{NMR}\left(400 \mathrm{MHz}, \mathrm{CDCl}_{3}\right) \delta$ : $1.64-1.71(1 \mathrm{H}, \mathrm{m}), 1.77-1.86(1 \mathrm{H}, \mathrm{m}), 1.90-1.99(2 \mathrm{H}, \mathrm{m}), 2.02-2.10$ $(1 \mathrm{H}, \mathrm{m}), 2.16-2.30(2 \mathrm{H}, \mathrm{m}), 2.31(3 \mathrm{H}, \mathrm{s}), 2.89-2.96(1 \mathrm{H}, \mathrm{m}), 3.75(1 \mathrm{H}, \mathrm{t}$ $J=6.0 \mathrm{~Hz}), 7.10(2 \mathrm{H}, \mathrm{d}, J=8.0 \mathrm{~Hz}), 7.31(2 \mathrm{H}, \mathrm{d}, J=8.0 \mathrm{~Hz}) \cdot{ }^{13} \mathrm{C}-\mathrm{NMR}$ $\left(150 \mathrm{MHz}, \mathrm{CDCl}_{3}\right) \delta: 21.1,22.5,27.3,33.8,38.9,57.0,129.8,129.9,132.7$, 137.8, 207.7. IR (neat): 2938, 1709, 1493, 1122, 1018, $809 \mathrm{~cm}^{-1}$. MS (EI) $m / z: 220\left(\mathrm{M}^{+}, 93 \%\right), 124\left(\mathrm{M}^{+}-\mathrm{C}_{6} \mathrm{H}_{8} \mathrm{O}, 100 \%\right)$. HR-MS Calcd for $\mathrm{C}_{13} \mathrm{H}_{16} \mathrm{OS}: 220.0922$. Found: 220.0918 .

2-(p-Chlorophenylthio)cyclohexanone ${ }^{1} \mathrm{H}-\mathrm{NMR}\left(400 \mathrm{MHz}, \mathrm{CDCl}_{3}\right) \delta$ $1.67-1.73(1 \mathrm{H}, \mathrm{m}), 1.79-1.96(3 \mathrm{H}, \mathrm{m}), 2.01-2.08(1 \mathrm{H}, \mathrm{m}), 2.19-2.32$ $(2 \mathrm{H}, \mathrm{m}), 2.85-2.92(1 \mathrm{H}, \mathrm{m}), 3.79(1 \mathrm{H}, \mathrm{t}, J=6.8 \mathrm{~Hz}), 7.25(2 \mathrm{H}, \mathrm{d}, J=8.8$ $\mathrm{Hz}), 7.33(2 \mathrm{H}, \mathrm{d}, J=8.8 \mathrm{~Hz}) .{ }^{13} \mathrm{C}-\mathrm{NMR}\left(100 \mathrm{MHz}, \mathrm{CDCl}_{3}\right) \delta: 22.6,27.2$, $33.8,39.0,56.5,129.1,132.2,133.2,133.6,207.2$. IR (neat): 2940,1710
1476, 1095, 1012, $826 \mathrm{~cm}^{-1}$. MS (EI) m/z: $240\left(\mathrm{M}^{+}, 69 \%\right), 144\left(\mathrm{M}^{+}-\right.$ $\left.\mathrm{C}_{6} \mathrm{H}_{8} \mathrm{O}, 100 \%\right)$. HR-MS Calcd for $\mathrm{C}_{12} \mathrm{H}_{13} \mathrm{ClOS}: 240.0376$. Found: 240.0357.

5-(m-Methoxyphenylthio)-6-undecanone ${ }^{21)}{ }^{1} \mathrm{H}$-NMR $\left(400 \mathrm{MHz}, \mathrm{CDCl}_{3}\right)$ $\delta: 0.85-0.91(6 \mathrm{H}, \mathrm{m}), 1.18-1.36(7 \mathrm{H}, \mathrm{m}), 1.43-1.47(1 \mathrm{H}, \mathrm{m}), 1.54(2 \mathrm{H}$, quint, $J=7.2 \mathrm{~Hz}), 1.67-1.75(1 \mathrm{H}, \mathrm{m}), 1.79-1.85(1 \mathrm{H}, \mathrm{m}), 2.56(2 \mathrm{H}, \mathrm{t}, J=$ $7.6 \mathrm{~Hz}), 3.65(1 \mathrm{H}, \mathrm{t}, J=7.6 \mathrm{~Hz}), 3.77(3 \mathrm{H}, \mathrm{s}), 6.78(1 \mathrm{H}, \mathrm{dd}, J=8.0,2.0 \mathrm{~Hz})$, $6.89-6.94(2 \mathrm{H}, \mathrm{m}), 7.19(1 \mathrm{H}, \mathrm{t}, J=8.0 \mathrm{~Hz}),{ }^{13} \mathrm{C}-\mathrm{NMR}\left(100 \mathrm{MHz}, \mathrm{CDCl}_{3}\right)$ $\delta: 13.8,13.8,22.3,23.5,29.4,30.1,31.2,39.1,55.1,56.8,113.4,116.9$, 123.9, 129.7, 134.7, 159.6, 207.7. IR (neat): 2956, 1590, 1282, 1249, 1043, $862,775,688 \mathrm{~cm}^{-1}$. MS (EI) $\mathrm{m} / z: 308\left(\mathrm{M}^{+}, 31 \%\right), 209\left(\mathrm{M}^{+}-\mathrm{C}_{6} \mathrm{H}_{11} \mathrm{O}\right.$, $100 \%$ ). HR-MS Calcd for $\mathrm{C}_{18} \mathrm{H}_{28} \mathrm{O}_{2} \mathrm{~S}: 308.1810$. Found: 308.1795 .

Acknowledgements This work was supported by Grant-in-Aid for Scientific Research (No. 21229001), GCOE program, and WPI Initiative from JSPS. M. A. expresses her thanks to the Grant-in-Aid for Scientific Research (No. 22689001) and the Asahi Glass Foundation.

\section{References}

1) Arisawa M., Yamaguchi M., J. Synth. Org. Chem. Jpn., 65, 12131224 (2007).

2) Arisawa M., Yamaguchi M., Pure Appl. Chem., 80, 993-1003 (2008).

3) Arisawa M., Fujimoto K., Morinaka S., Yamaguchi M., J. Am. Chem. Soc., 127, 12226-12227 (2005).

4) Arisawa M., Kubota T., Yamaguchi M., Tetrahedron Lett., 49, 19751978 (2008).

5) Grossert J. S., Dubey P. K., Chem. Commun., 1982, 1183-1184 (1982).

6) Tsunetsugu J., Ikeda T., Suzuki N., Yaguchi M., Sato M., Ebine S., Morinaga K., J. Chem. Soc., Perkin 1, 1985, 785-794 (1985).

7) Trost B. M., Mao M. K.-T., Balkovec J. M., Buhlmayer P., J. Am. Chem. Soc., 108, 4965-4973 (1986).

8) Sugihara Y., Wakabayashi S., Saito N., Murata I., J. Am. Chem. Soc., 108, 2773-2775 (1986).

9) Woodward R. B., Pachter I. J., Scheinbaum M. L., J. Org. Chem., 36, 1137-1139 (1971)

10) Trost B. M., Hiroi K., Jungheim L. N., J. Org. Chem., 45, 1839-1847 (1980).

11) Imoto S., Haruta Y., Watanabe K., Sasaki S., Bioorg. Med. Chem. Lett., 14, 4855-4859 (2004).

12) Tosaka A., Ito S., Miyazawa N., Shibuya M., Ogasawara K., Iwabuchi Y., Heterocycles, 70, 153-159 (2006).

13) Padmanabhan S., Ogawa T., Suzuki H., Bull. Chem. Soc. Jpn., 62, 1358-1360 (1989).

14) Arisawa M., Suwa K., Yamaguchi M., Org. Lett., 11, 625-627 (2009).

15) Reese C. B., Sanders H. P., J. Chem. Soc., Perkin Trans. 1, 1982, 2719-2724 (1982)

16) Tehrani K. A., Boeykens M., Tyvorskii V. I., Kulinkovich O., De Kimpe N., Tetrahedron, 56, 6541-6548 (2000).

17) Reich H. J., Renga J. M., Reich I. L., J. Am. Chem. Soc., 97, 54345447 (1975).

18) Matsugi M., Murata K., Gotanda K., Nambu H., Anilkumar G., Matsumoto K., Kita Y., J. Org. Chem., 66, 2434-2441 (2001).

19) Ishibashi H., Takamuro I., Mizukami Y., Irie M., Ikeda M., Synth. Commun., 1989, 443-452 (1989).

20) Loghmani-Khouzani H., Poorherva M. R., Sadeghi M. M. M., Caggiano L., Jackson R. F. W., Tetrahedron, 64, 7419-7425 (2008).

21) Oliveira M. M., Moustrou C., Carvalho L. M., Silva J. A. C., Samat A., Gugliemetti R., Dubest R., Aubard J., Oliveira-Campos A. M. F., Tetrahedron, 58, 1709-1718 (2002). 